\title{
Status of the Regenerative ECLS Water Recovery System
}

\author{
Layne Carter ${ }^{1}$ \\ NASA Marshall Space Flight Center, Huntsville, AL 35812
}

\begin{abstract}
The regenerative Water Recovery System (WRS) has completed its first full year of operation on the International Space Station (ISS). The major assemblies included in this system are the Water Processor Assembly (WPA) and Urine Processor Assembly (UPA). This paper summarizes the on-orbit status as of May 2010, and describes the technical challenges encountered and lessons learned over the past year.
\end{abstract}

\section{Introduction}

$\mathrm{T}$

he U.S. regenerative Environmental Control and Life Support Systems (ECLSS) have been developed to enable the ISS to expand its crew size from three to six crewmembers. Regenerative ECLS includes the Water Recovery System (WRS), comprised of the Urine Processor Assembly (UPA) and Water Processor Assembly (WPA). This hardware produces potable water from a combination of condensate and urine collected on ISS. The Water Recovery System is located in two ISPR racks, named WRS\#1 and WRS\#2. This hardware was delivered to ISS on STS-126 on November 14, 2008 and initially installed in the US Lab module. On February 18, 2010, the racks were transferred to their permanent home in the Node 3.

\section{Description of the ISS Water Recovery System}

The layout of the two WRS racks is shown in Figure 1, along with the Oxygen Generation System (OGS). The WPA is packaged entirely in WRS rack \#1 and partially in WRS Rack \#2, linked by process water lines running between the two racks. The remaining portion of WRS Rack \#2 houses the UPA.

The following sections provide a description of the WRS, give current status, and describe issues and lessons learned during the past year. For the prior years' status, see references 1-2.

\footnotetext{
${ }^{1}$ Life Support Development Team Lead, NASA MSFC ES62.
} 


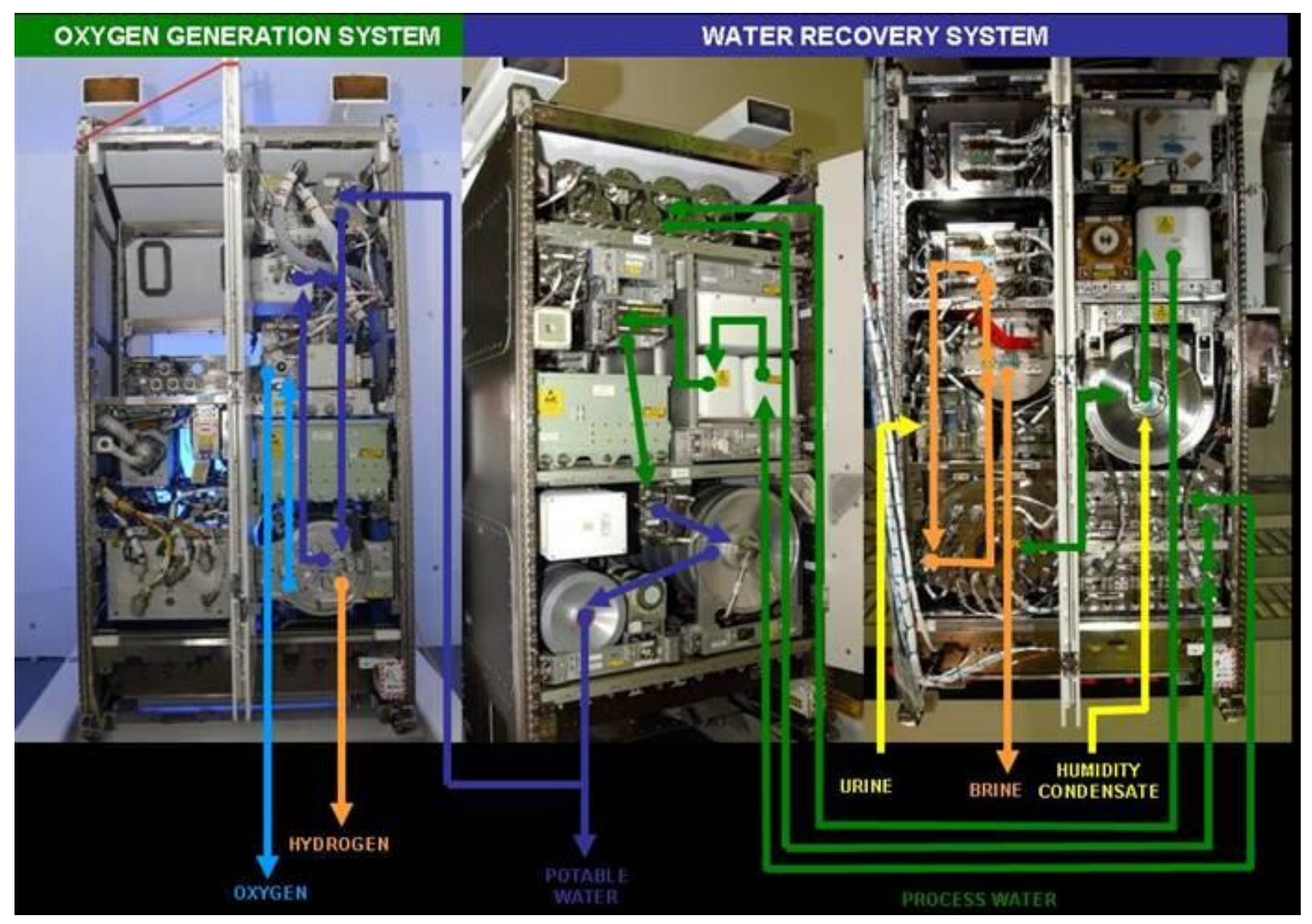

\section{FIGURE 1. INTERNATIONAL SPACE STATION REGENERATIVE ECLSS}

\section{A. Water Processor Assembly Overview}

A simplified schematic of the WPA is provided in Figure 2. Wastewater delivered to the WPA includes condensate from the Temperature and Humidity Control System and distillate from the UPA. This wastewater is temporarily stored in the Waste Water Tank Orbital Replacement Unit (ORU). The Waste Water Tank includes a bellows that maintains a pressure of approximately $5.2-15.5 \mathrm{kPa}(0.75$ to $2.25 \mathrm{psig})$ over the tank cycle, which serves to push water and gas into the Mostly Liquid Separator (MLS). Gas is removed from the wastewater by the MLS (part of the Pump/Separator ORU), and passes through the Separator Filter ORU where odor-causing contaminants are removed from entrained air before returning the air to the cabin. Next, the water is pumped through the Particulate Filter ORU followed by two Multifiltration (MF) Beds where inorganic and non-volatile organic contaminants are removed. Once breakthrough of the first bed is detected, the second bed is relocated into the first bed position, and a new second bed is installed. The Sensor ORU located between the two MF beds helps to determine when the first bed is saturated based on conductivity. Following the MF Beds, the process water stream enters the Catalytic Reactor ORU, where low molecular weight organics not removed by the adsorption process are oxidized in the presence of oxygen, elevated temperature, and a catalyst. A regenerative heat exchanger recovers heat from the catalytic reactor effluent water to make this process more efficient. The Gas Separator ORU removes excess oxygen and gaseous oxidation by-products from the process water and returns it to the cabin. The Reactor Health Sensor ORU monitors the conductivity of the reactor effluent as an indication of whether the organic load coming into the reactor is within the reactor's oxidative capacity. Finally, the Ion Exchange Bed ORU removes dissolved products of oxidation and adds iodine for residual microbial control. The water is subsequently stored in the product water tank prior to delivery to the ISS potable water bus. The Water Delivery ORU contains a pump and small accumulator tank to deliver potable water on demand to users. The WPA is controlled by a firmware controller. 


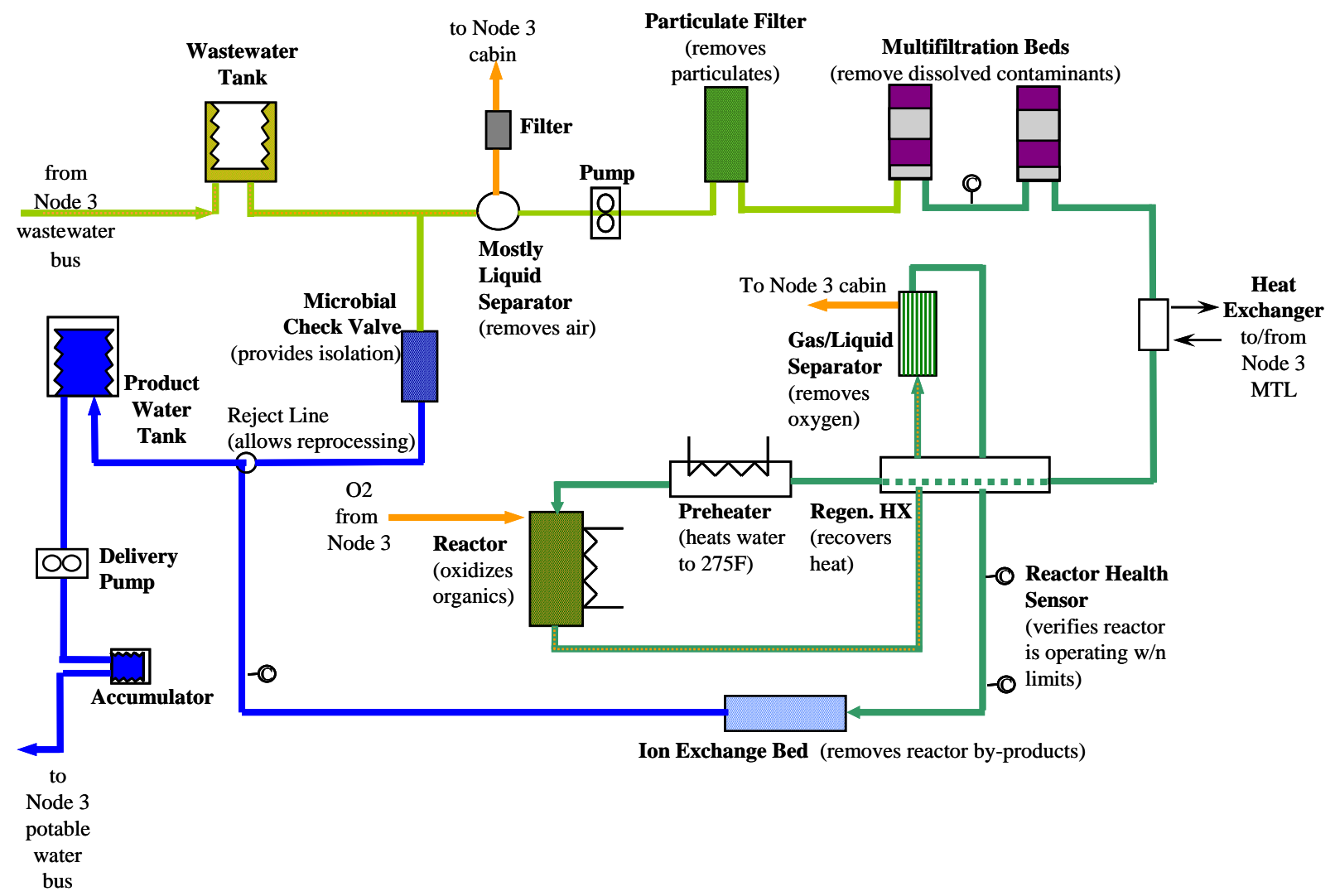

Figure 2. WPA Simplified Schematic

\section{B. Urine Processor Assembly Overview}

A simplified schematic of the UPA is shown in Figure 3. Pretreated urine is delivered to the UPA either from the USOS Waste and Hygiene Compartment (outfitted with the Russian urinal) or it can be supplied via manual transfer from the Russian urine container (called an EDV). In either case, the composition of the pretreated urine is the same, including urine, flush water, and a pretreatment formula containing chromium trioxide and sulfuric acid to control microbial growth and the reaction of urea to ammonia. The urine is temporarily stored in the Wastewater Storage Tank Assembly (WSTA). When a sufficient quantity of feed has been collected in the WSTA, a process cycle is automatically initiated. The Fluids Control and Pump Assembly (FCPA) is a four-tube peristaltic pump that moves urine from the WSTA into the Distillation Assembly (DA), recycles the concentrated waste from the DA into the Recycle Filter Tank Assembly (RFTA) and back to the DA, and pumps product distillate from the DA to the wastewater interface with the WPA. The DA is the heart of the UPA, and consists of a rotating centrifuge where the waste urine stream is evaporated at low pressure, compressed, and subsequently condensed on the opposite side of the evaporator surface to conserve latent energy. A rotary lobe compressor provides the driving force for the evaporation and compression of water vapor. Waste brine resulting from the distillation process is stored in the RFTA, which has a capacity of approximately $41 \mathrm{~L}$. When the brine is concentrated to the required limit, the RFTA is replaced with an empty RFTA, which allows the process to repeat. The full RFTA is returned to the ground on the Shuttle for refurbishment so it can be returned to ISS for another cycle. The Pressure Control and Pump Assembly (PCPA) is another four-tube peristaltic pump which provides for the removal of non-condensable gases and water vapor from the DA. Liquid cooling of the pump housing promotes condensation, thus reducing the required volumetric capacity of the peristaltic pump. Gases and condensed water are pumped to the Separator Plumbing Assembly (SPA), which recovers and returns water from the purge gases to the product water stream. A Firmware Controller Assembly (FCA) provides the command control, excitation, monitoring, and data downlink for UPA sensors and effectors. 
The UPA was designed to process a nominal load of $9 \mathrm{~kg} /$ day $(19.8 \mathrm{lbs} /$ day) of wastewater consisting of urine and flush water. This is the equivalent of a 6-crew load on ISS. Product water from the UPA must meet specification quality requirements for conductivity, $\mathrm{pH}$, ammonia, particles, and total organic carbon. The UPA was designed to recover $85 \%$ of the water content from the pretreated urine, though issues encountered in the last year have required the $\%$ recovery to be dropped to $70 \%$.

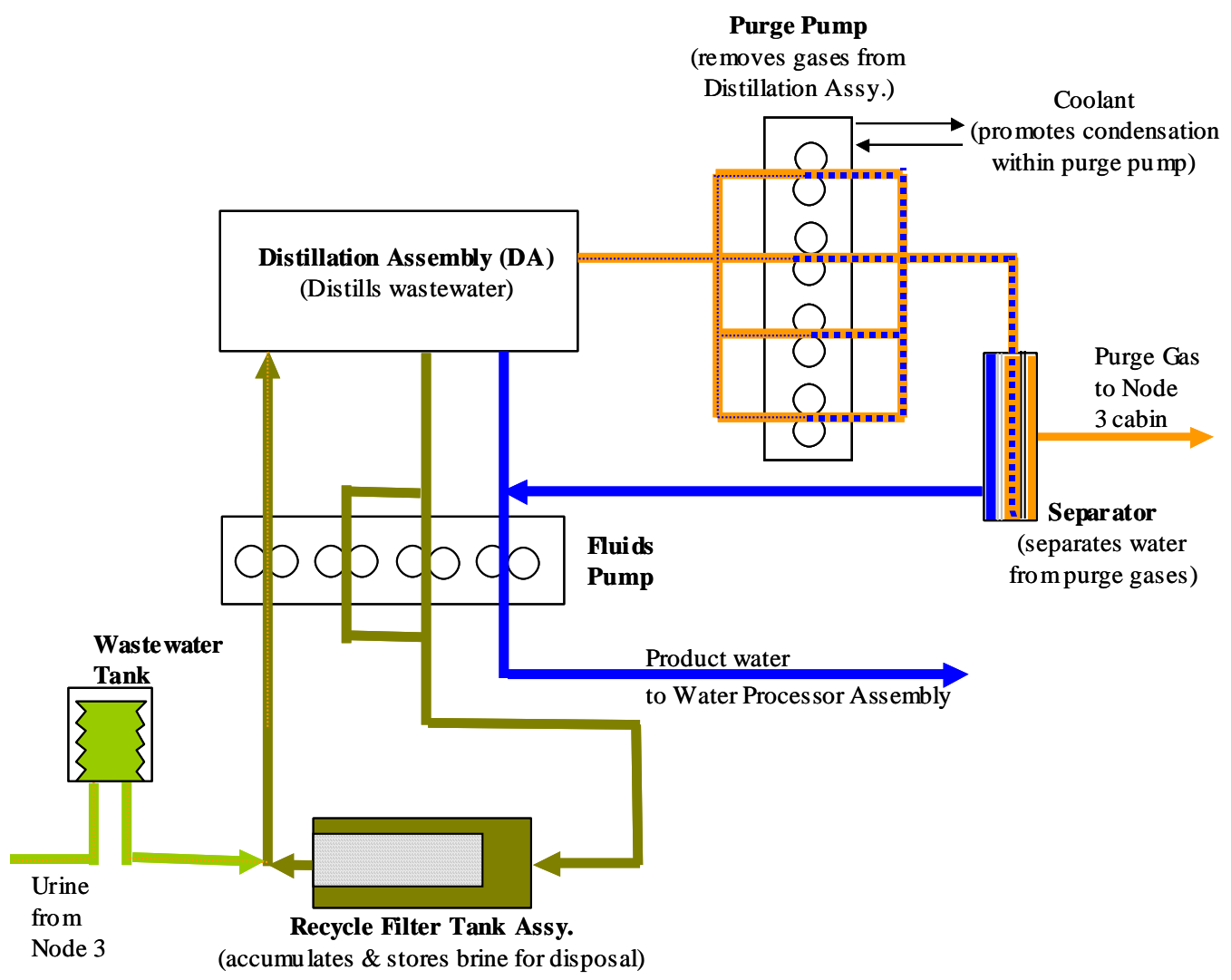

Figure 3. Urine Processor Assembly Schematic

The UPA is packaged into 7 ORUs, which take up slightly more than half of the WRS Rack \#2. The RFTA is the only expendable ORU, sized for a 30-day replacement schedule when processing the daily urine load from 6 crewmembers.

\section{Urine Processor Assembly Current Status}

The UPA was initially activated on November 20, 2008. As of May 2010, the UPA has processed 2270 L (5000 lb) of pretreated urine, producing $1740 \mathrm{~L}(3850 \mathrm{lb})$ of distillate and cycling through 14 RFTAs during that time. Operation of the UPA on ISS has been interrupted by two separate failures of the Distillation Assembly.

As discussed in the previous paper (1), DA S/N 01 failed in December 2008 after processing $180 \mathrm{~L}(400 \mathrm{lb})$ of pretreated urine and was replaced with S/N 02 during the 15A mission on March 20, 2009. This failure was manifested on ISS as a high current fault of the DA motor. The DA was returned to the Marshall Space Flight Center (MSFC) for a subsequent failure investigation. This investigation verified that the centrifuge had contacted 2 of the 3 speed sensors used to monitor centrifuge speed, though the high current was due to contact between the compressor lobes and the compressor endplate. Subsequent analyses confirmed the interference with the speed sensors was due to a number of issues. Thermal growth and the vacuum maintained in the DA will result in closing this gap, though this mechanical tolerance was accounted for in the initial design of the hardware. However, during the failure investigation it was determined that one of the speed sensors was not correctly installed following a failure during ground tests at MSFC of the UPA. As a result, the speed sensor was located $0.043 \mathrm{~cm}(0.017$ inches) closer to the centrifuge than specified. Also, the front bearing press (locating the centrifuge on the main shaft) did not adequately capture the bearing, thus allowing slight axial movement (approximately $0.01 \mathrm{~cm}$ ) of the centrifuge. 
Finally, the DA was initially soft-mounted in the rack for operation on ISS (to reduce acoustic emissions), which allowed mechanical vibration to be accentuated. These issues all contributed to the contact between the speed sensor and the centrifuge. However, it cannot be determined if this contact was ultimately responsible for the mechanical interference in the compressor. The root cause for the compressor lobes contacting the end plate was a structural failure of the compressor's pinion bearings. This failure may have occurred during assembly of the DA, during launch of the DA to ISS, or due to the loads that occurred on ISS when the centrifuge contacted the speed sensor.

To address the structural failure of the pinion bearings, these bearings have been replaced with those made of a harder material that will withstand the loads that damaged the failed bearings. Though root cause cannot be determined, the stress analysis of the new bearings has confirmed that they can sustain the worst-case loads experienced over the life of the DA. In addition, new procedures are in place during DA assembly to insure the structural load on the bearing does not exceed specification for the bearings. To address the contact between the centrifuge and speed sensor, design modifications have been implemented to insure adequate bearing retention for the centrifuge on the main shaft. Also, installation of the speed sensors will only be performed per standard procedure to insure the required distance from the centrifuge is maintained. Finally, the DA will always be hardmounted in WRS\#2 on ISS to dampen any vibration affects associated with DA operation.

A separate anomaly also discussed in the previous paper (1) was the failure of the FCPA check valve. The purpose of this check valve is to prevent backflow from the WPA waste tank into the DA. On April 232009 , the check valve failed closed during normal operation of the fluids pump, and repeated attempts to open it by operating the pump were unsuccessful. Since this check valve is a redundant control against backflow (and not required as a safety control), engineering personnel determined that it should be removed from the hardware on ISS. This allowed for the first intermediate-level (I-level) activity on UPA. I-level refers to on-orbit maintenance of hardware internal to an ORU, which avoids the costly effort to return and resupply an entire ORU on ISS that has failed due to a single component. The check valve is an inline component in the FPCA manifold (see Figure 4). Due to this design, the valve could be removed by first removing the inlet flex hose/cap to the manifold, then the check valve, and finally replacing the flex hose. Implementation of this procedure was challenging because the required tools were not available on ISS, requiring creative solutions by the ISS engineering/operations personnel. The flex hose/cap (hose not shown in Figure 4) is held in place with two fasteners. After the fasteners were removed, the cap was removed by gently pulling on the base of the hose with a pair of pliers to overcome the resistance of the two radial o-rings. The inline check valve is held in place by a separate radial o-ring. Threads at the inlet to the check valve are provided for removal, but require a bolt with the mating threads (not present on ISS). Instead, operations personnel identified a plastic nozzle from a foam applicator available on ISS with threads slightly smaller than the required bolt. These threads were then wrapped with Kapton tape to better match the threads in the check valve (see Figure 5). Despite the use of the modified hardware as tools, the crew was successfully able to remove the check valve and therefore return the UPA to operation.

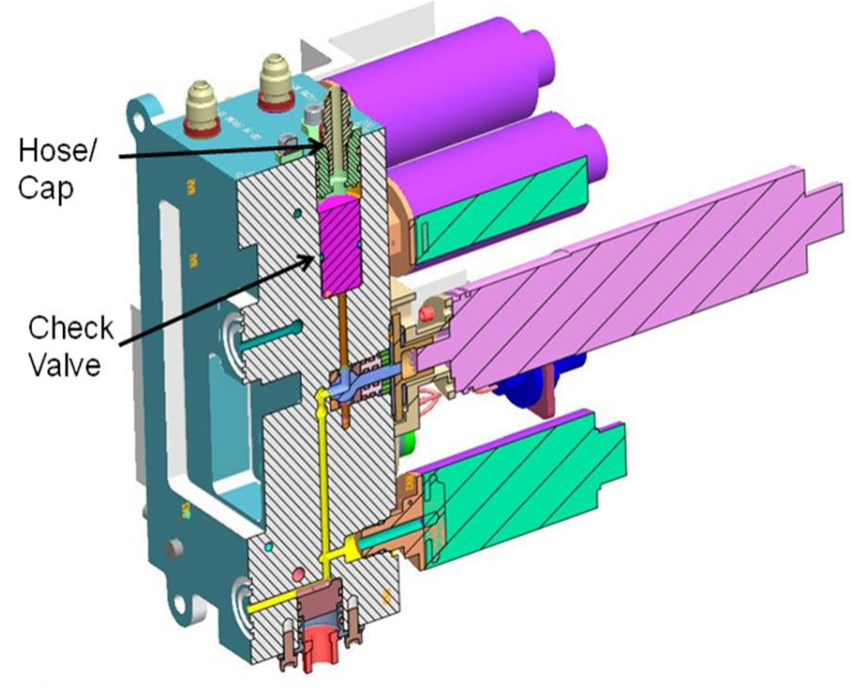

Figure 4. FCPA Manifold

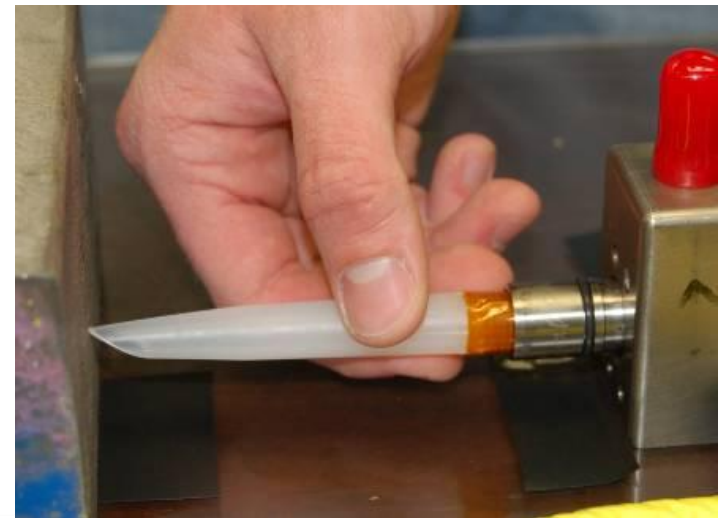

Figure 5. Removal of Check Valve (ground demo) 
DA S/N 02 was operational on ISS from March 20, 2010 to October 24, 2010, during which time the UPA processed $1320 \mathrm{~L}(2900 \mathrm{lb})$ of pretreated urine and produced approximately $1120 \mathrm{~L}(2470 \mathrm{lb})$ of distillate. On October 24, 2010, the DA S/N 02 experienced a high current fault in the compressor. After a review of the telemetry data, it was observed that the UPA waste tank had decreased in quantity by approximately $4.5 \mathrm{~L}(10 \mathrm{lb})$ during a process cycle, while there was no corresponding increase in the WPA waste tank quantity. The most likely reason for this imbalance is due to flooding of the DA. The UPA was first commanded to perform an additional drydown process, which serves to pump brine out of the DA and into the UPA Waste Tank. This procedure was initially successful (allowing UPA to return to a normal process cycle), but the UPA flooded again during the next process cycle. Subsequent procedures were unsuccessful in removing any significant quantity of brine out of the DA. To address the possibility that solids had formed in the brine pitot tube (that removes brine from the DA), the crew reconfigured the UPA plumbing such that the Fluids Pump could flow a small volume of brine backwards through the pitot tube and into the DA. Subsequently, another drydown process was attempted, with limited success. At this point, it was determined the DA could not be recovered, and the decision was made to return it to the ground for a failure investigation.

After return to the Marshall Space Flight Center in December 2009, the DA was disassembled. The root cause of the anomaly was found in the evaporator, which contained a significant quantity of solids (see Figure 6). These solids had blocked the brine pitot tube, resulting in flooding the DA. In addition, the urine brine had passed through the demister and the compressor lobes, and was found in the DA's condenser and the stationary bowl.

Subsequent analysis of the solids determined that they were primarily comprised of calcium sulfate. Calcium is present in the urine primarily due to bone loss from the crew, whereas sulfate is present primarily due to the use of sulfuric acid in the urine pretreatment. Calcium levels on ISS are elevated compared to ground urine due to the absence of gravity. During ground testing, the UPA was proven to have no issues with recovering $85 \%$ of the water from pretreated urine. However, at $85 \%$ recovery on ISS, the higher concentration of calcium resulted in calcium sulfate exceeding its solubility limit. Since calcium sulfate solubility is lower at elevated temperature, it precipitated in the DA where the fluid is at the maximum temperature in the recycle loop.

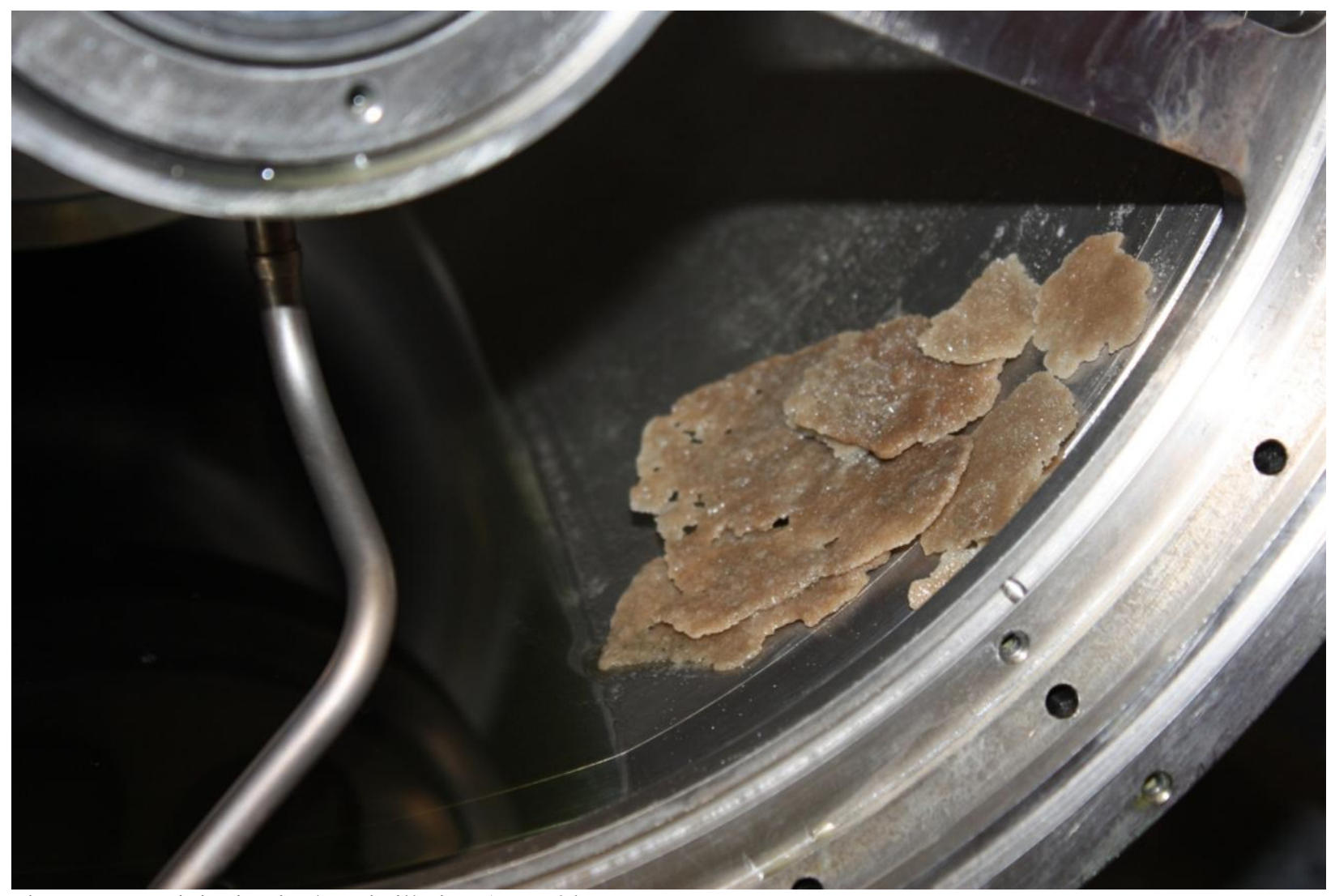

Figure 6. Precipitation in the Distillation Assembly Evaporator 
In addition to the elevated calcium concentration, data from pretreated urine and brine has shown an elevated $\mathrm{pH}$ level. During ground tests, the urine $\mathrm{pH}$ was typically 1.5 to 1.8 , and the brine $\mathrm{pH}$ would drop to approximately 1.1 to 1.4. However, the $\mathrm{pH}$ of the urine and brine on ISS has ranged from 1.7 to 2.5 . This elevated $\mathrm{pH}$ is an issue because lower $\mathrm{pH}$ levels inhibit precipitation of the calcium sulfate. In addition, elevated $\mathrm{pH}$ levels can result in a loss of microbial control in the brine loop, though fortunately no microbial content was detected in analysis of urine and brine that has been returned to the ground. The reason for the elevated $\mathrm{pH}$ level is not specifically known, but it does appear to correlate with elevated phosphate levels in the urine.

Based on these observations, engineering personnel agreed that $85 \%$ recovery of water from the pretreated urine was not viable. A rigorous statistical analysis of urine chemistry was performed by personnel at the Johnson Space Center (JSC) and MSFC using analysis of samples taken on ISS. This analysis defined a $90 \%$ upper confidence limit on the calcium concentration as $283 \mathrm{mg} / \mathrm{L}$. Given this concentration, a $70 \%$ recovery has been specified for future operation of the UPA, which accepts an $8 \%$ risk of reaching the calcium solubility limit over a given crew increment. Though this approach has some risk, $70 \%$ recovery was deemed necessary to meet logistics requirements for RFTA refurbishment and water resupply. In parallel with this modification to on-orbit operations, NASA personnel will investigate options for either removing calcium from the urine prior to introduction to the UPA, or introducing chelating agents into the pretreated urine that will inhibit precipitation of the calcium sulfate.

In addition, the rebuild of the DA S/N 02 will incorporate modifications to the demister. Water vapor generated by the evaporator flows through the demister before entering the compressor. The function of the demister is to collect liquid droplets in the vapor stream and sling them back into the evaporator. The investigation of the failed DA determined that the demister was not effective in removing liquid from the vapor stream, resulting in precipitation of solids on an inlet screen to the compressor. This ultimately caused elevated compressor temperature when the DA flooded and the inlet screen was almost completely blocked with precipitation. The demister redesign will address deficiencies in the demister functions to insure liquid droplets are effectively collected and returned to the evaporator, thus protecting the compressor from ingesting liquid.

DA S/N 01 was refurbished at MSFC and returned to ISS in February 2010. Pending the completion of the failure investigation of S/N 02, the first 5 RFTAs were only processed to $65 \%$ recovery, after which the next RFTA (and all subsequent RFTAs) was processed to 70\% recovery. The UPA has currently processed $770 \mathrm{~L}$ (1700 lb) of pretreated urine with DA S/N 01 in 2010, producing $500 \mathrm{~L}(1090 \mathrm{lb})$ of distillate.

\section{Water Processor Assembly Current Status}

The WPA was initially activated on November 22, 2008. As of May 12, 2010, the WPA has processed approximately $3970 \mathrm{~kg}(8760 \mathrm{lb})$ of waste water. There is limited water quality data available for the last year of WPA performance, since all samples returned to ground are taken from the Potable Water Dispenser (PWD). Though water for the dispenser comes from the WPA, the WPA product water is processed through a small bed of ion exchange resin and adsorbent for removal of iodine, therefore the data is not necessarily representative of the WPA product water. However, on-orbit samples are taken of the WPA for subsequent analysis by the Total Organic Carbon Analyzer (TOCA). These results have all been reported less than the TOCA detection limit of $0.475 \mathrm{mg} / \mathrm{L}$, confirming that the WPA is continuing to produce high quality water. After the initial certification of the WPA was completed in early 2009, the on-orbit Water Microbial Kit (WMK) is also typically only used to assess the microbial count delivered by the PWD. Since these results are impacted by the microbial contamination internal to the PWD, they are not relevant to the WPA performance. However, after installation of Catalytic Reactor S/N 02 in March 2010, an on-orbit microbial analysis was performed of the WPA product water to confirm the WPA was producing water meeting the microbial specification of 50 Colony Forming Units $(\mathrm{CFU}) / \mathrm{ml}$. The result was $3 \mathrm{CFU} / \mathrm{ml}$, confirming the performance of the WPA.

Two anomalies have occurred to the WPA in the past year on ISS. First, the WPA began exhibiting signs of increased pressure drop between the waste tank and the MLS in June 2009. This trend was based on a decreased pressure at the MLS inlet pressure, and increased pressure drop downstream of the process pump. At the time, this appeared to be a transient and was not identified as an issue until September 2009, when the condition worsened to the point that the flow from the waste tank to the MLS became so low that the MLS could not properly feed the pump with waste water. Typically there is sufficient static pressure from the waste water tank to maintain a higher flow rate to the MLS than the rate at which the pump empties the MLS $(5.9 \mathrm{~kg} / \mathrm{hr}, 13 \mathrm{lb} / \mathrm{hr})$. However, at lower tank quantities, the pressure drop between the waste tank and the MLS reduced the flow to a level below the pump's nominal flow rate, resulting in the MLS draining to its minimum setting. When the fluid level in the MLS reaches this setting, the software initiates a Purge and Reprocess cycle (to prevent gas from being fed to the pump). 
To support continued WPA operations, the WPA was operated in a "degraded" mode. The primary modification in this mode was to operate the WPA at a reduced flow rate, which allowed more time for the waste tank to fill the MLS. This flow rate was approximately $4.5 \mathrm{~kg} / \mathrm{hr}(10 \mathrm{lb} / \mathrm{hr})$, and was established slightly above the minimum flow rate required to maintain a temperature delta of $2.8 \mathrm{C}(5 \mathrm{~F})$ across the Gas/Liquid Separator (GS). This was necessary because excessive temperature variance across the GS can result in condensation in the sweep air, which would render the GS inoperable. This degraded mode of operations allowed the WPA to continue delivery of product water until January 2010. At this point, an attempt was made to reverse flush the plumbing between the waste water tank and the MLS by reconfiguring the plumbing and using an external pump to flow water through the lines at $0.5 \mathrm{~L} / \mathrm{min}$. Though there was an initial reduction in the pressure drop for one process cycle, the WPA failed on January 12, 2010 when there was no measurable flow of water from the waste tank to the MLS. The Pump/Sep ORU S/N 01 was replaced with the spare unit (S/N 02) later in January. Prior to beginning WPA operations, however, an additional filter (delivered on Shuttle Endeavor in February 2010) was installed upstream of the Pump/Sep ORU to protect the clearances in the MLS inlet solenoid valve. This valve was identified to contain the tightest clearance in the line between the waste tank and the MLS, and as such was the suspected location of the increased pressure drop. This filter allowed WPA operations to begin on February 11, 2010. The failed unit was returned to the ground on Shuttle Endeavor for a failure investigation.

The failed ORU was disassembled at Hamilton Sundstrand the week of February 22, 2010. Though the plumbing internal to the ORU exhibited no significant indication of biofouling, the MLS inlet solenoid valve was found to be significantly occluded with biomass. This biomass had accumulated in the valve between the poppet and the valve seat, obstructing the clearance in this region and causing the excessive pressure drop. Figure 7 provides a view of section of the valve that feeds the poppet, illustrating the accumulation of biomass in all but one channel.

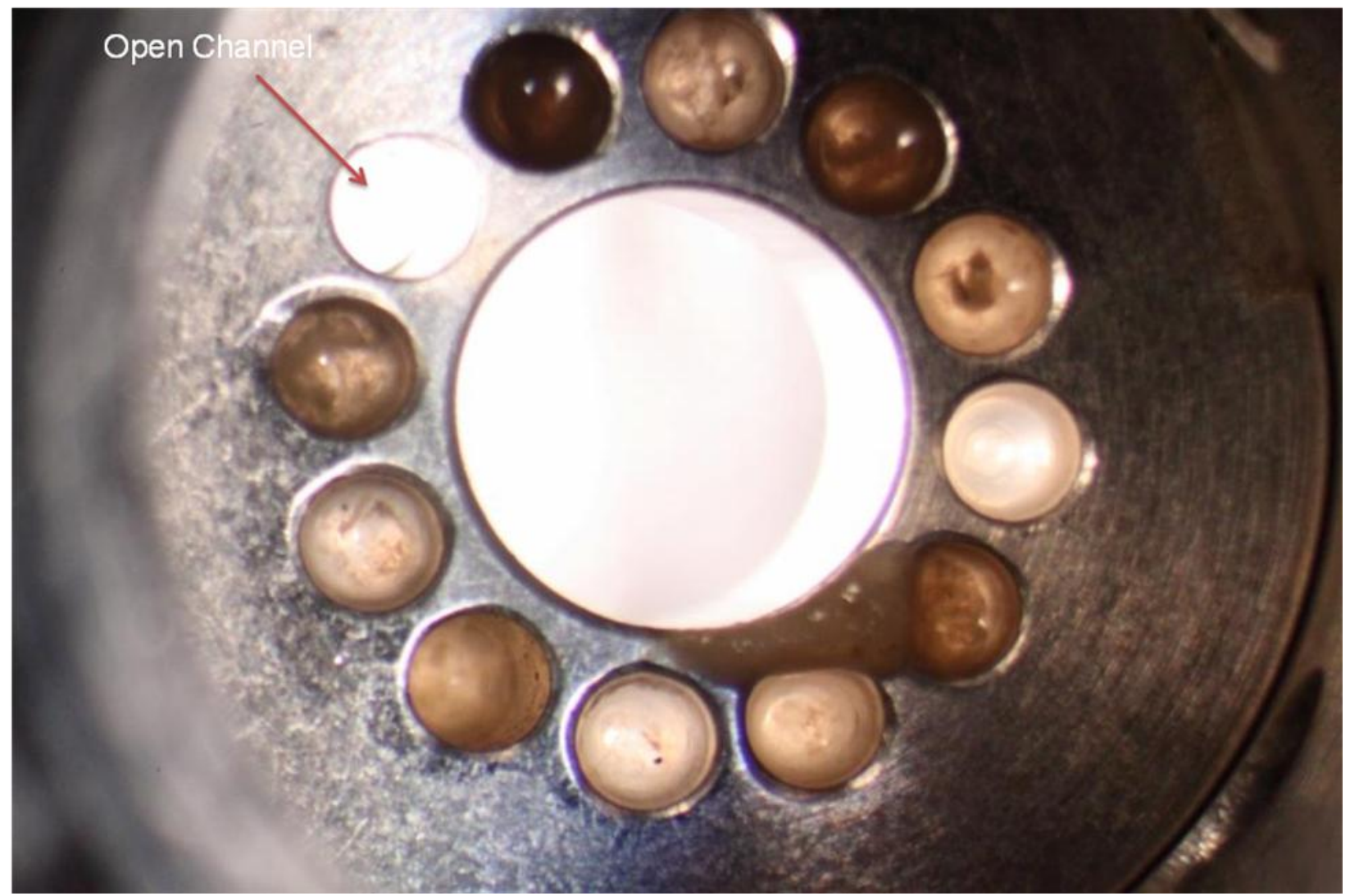

Figure 7. Biomass Present in MLS Inlet Solenoid Valve

Based on this investigation, engineering personnel are pursuing multiple efforts to insure the continued operation of the WPA on ISS. First, additional filters have been shipped to ISS as spares for the filter currently installed upstream of the MLS. Filter life is being tracked to determine if this approach is viable for long term WPA operation. Currently, after two months of operation on ISS, no significant increase in pressure drop has been 
observed. If the current filter life is deemed unacceptable, a modified filter design will be delivered to ISS that provides additional filter surface area. Also, an additional solenoid valve may be built and delivered to ISS to be replaced by the crew if the failure occurs again.

The second WPA anomaly occurred in March 2010 only a few weeks after the Pump/Sep ORU was replaced. After the WPA operations were initiated following installation of the new Pump/Sep ORU, engineering personnel observed that the process pump was cycling more than usual to maintain pressure in the Catalytic Reactor. On March 22, 2010, a failure occurred due to low temperature in the reactor's preheater. While investigating this failure, engineering personnel observed that the waste tank quantity was continuously decreasing while the pump was pressurizing the reactor, indicating a leak in the system. After completing troubleshooting steps to isolate the leak to the Catalytic Reactor ORU, a crew inspection identified the leak in the end of 3 of the 4 reactor tubes. This leak occurred through a standard fitting, but the fact that 3 of the 4 tubes were leaking indicated a systemic issue either with the fitting design or its application.

The spare Catalytic Reactor ORU S/N 02 was delivered to ISS on Shuttle Discovery in April 2010 and installed in the WPA on April 11, 2010. The failed Catalytic Reactor ORU S/N 01 was returned on the same Shuttle for a subsequent failure investigation. After initially completing a Computerized Tomography (CT) scan at MSFC to confirm the configuration of the seals in the reactor tube fittings, the hardware investigation was completed at Hamilton Sundstrand. An initial leak test of the reactor confirmed the location of the leaks identified on ISS, and also identified an additional leak at the other end of the reactor tube. All leaks occurred through a standard MS Boss fitting, a cross section of which can be found in Figure 8. This fitting uses an o-ring made of ethylene propylene diene monomer (EPDM), which is the optimum rubber to be used in aqueous applications at temperatures above 65 $\mathrm{C}$ (Catalytic Reactor operating temperature is $130 \mathrm{C}$ ). In an ideal application, the o-ring is pushed by the jam nut and washer into the annulus between the female bore and the male gland as shown in Figure 8. Figure 9 shows an oring from an ambient temperature application in the Catalytic Reactor ORU after 8 years in place. As expected, the o-ring has slightly deformed to fill the annulus and thus provide the seal against external leakage.

\section{EPDM o-ring}

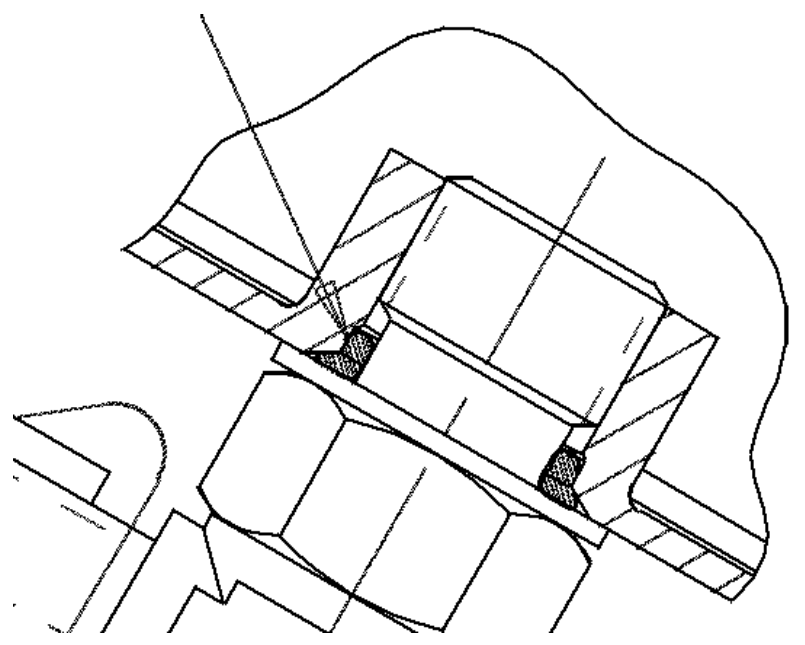

Figure 8. Standard MS Boss Fitting

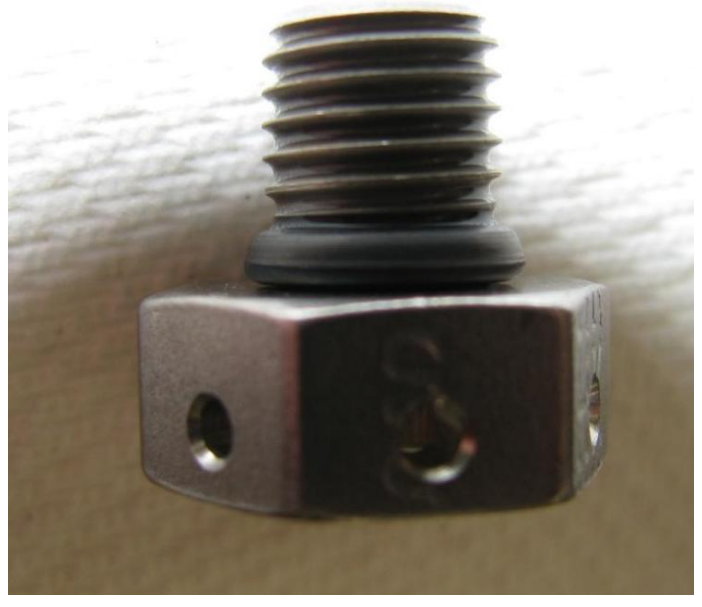

Figure 9. View of Ambient Temperature O-ring

However, the o-ring selected for this application did not take into account expansion of the o-ring due to swell (water absorption) or thermal expansion at the elevated temperature application. Therefore, the o-ring volume exceeded the available gland volume at elevated temperature, ultimately resulting in compression set. Figure 10 shows the fitting from the $3^{\text {rd }}$ reactor tube, which was the location for the highest leak rate. In addition to the compression set (note the o-ring was deformed to the available gland volume), this o-ring had also been installed over the bottom thread. Also, the o-ring had even extruded through the inside diameter of the washer because of the fact that it exceeded the available gland volume at the elevated temperature.

To support refurbishment of Catalytic Reactor ORU S/N 01, the hardware is being redesigned to eliminate the overfill condition of the o-ring in the gland at the elevated temperature. This is accomplished with some combination of modifications, including an alternate EPDM o-ring with less swell, a custom o-ring with a smaller 
cross section, and/or a machined fitting with a smaller diameter. The objective for the refurbishment of this ORU is to return it to ISS on Shuttle Endeavor in October 2010 if the schedule permits.

Other than the replacement of the Pump/Sep ORU and the Catalytic Reactor ORU, no other items have been replaced in the WPA. As of May 2010, there are no indications that the Particulate Filter or Multifiltration Bed is loading. Scheduled replacement of the Sep Filter ORU and Ion Exchange Bed ORU has not yet occurred. Though these items were scheduled for annual replacement, this requirement was based on the expected throughput (per the ISS WRS Specification). Actual throughput on ISS is less than the quantity defined in the WRS requirements, therefore the actual life for these items has been extended based on the actual throughput.

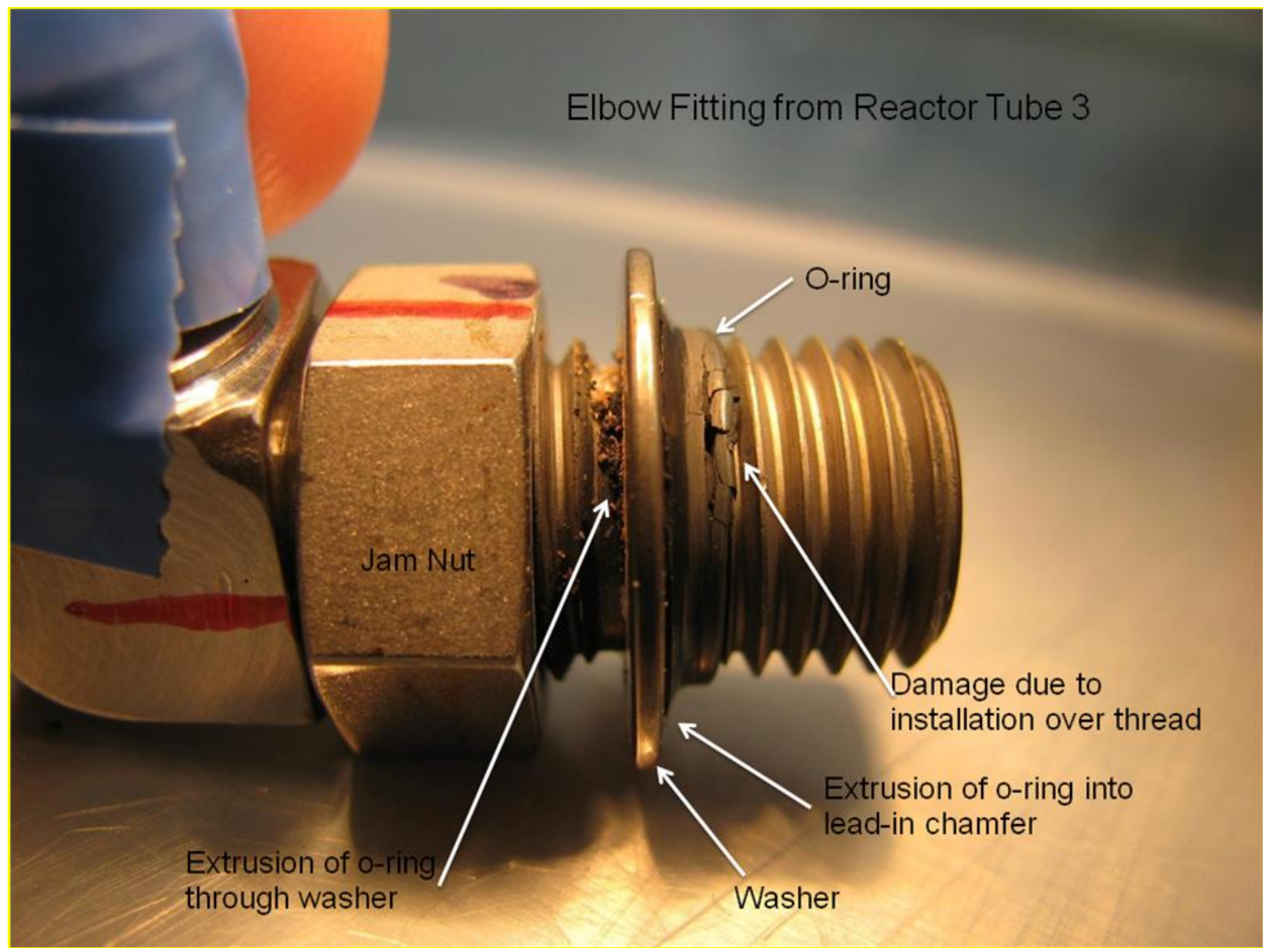

Figure 10. View of Damaged O-ring

\section{Conclusion}

In the past year, the WRS has continued to provide the ISS crew with potable water for drinking, electrolysis via the Oxygen Generation System, flush water for the Waste \& Hygiene Compartment, and hygiene water. However, the WPA and UPA have experienced several operational and design issues. The DA anomaly observed initially in 2008 was resolved with the implementation of harder compressor bearings and a redesign to the demister, while the precipitation issue is currently handled by reducing the urine recovery to $70 \%$ instead of $85 \%$. In parallel, NASA personnel will investigation options to achieve higher \% recovery by removal of calcium or inhibiting calcium sulfate precipitation.

The WPA failure of the MLS inlet solenoid valve is due to biomass obstruction of the MLS inlet solenoid valve, which is currently addressed through the addition of a filter upstream of the MLS. The leak in the Catalytic Reactor ORU was due to a design flaw in the MS Boss fitting, and will be resolved with improved seal selection for the refurbishment of S/N 01. The subsequent design of this ORU may incorporate the same seal design, or an altered fitting may be developed that does not require an o-ring, thus avoiding the sensitivity to operation at the elevated temperature. 


\section{Acknowledgments}

The author wishes to acknowledge the effort of the many engineers at NASA MSFC/JSC, Hamilton Sundstrand, and Boeing that have performed excellent work in the last year toward the operation, troubleshooting, and recovery of the Water Recovery System hardware on ISS.

\section{References}

1. Carter, D.L., "Status of the Regenerative ECLSS Water Recovery System", SAE 2000-01-2352, presented at the 39"th International Conference on Environmental Systems, Savannah, Georgia, July, 2009.

2. Bagdigian, R.M., D.L. Carter, and G. Sitler, "Status of the Regenerative ECLSS Water Recovery System", SAE 2008-012133, presented at the $38^{\text {th }}$ International Conference on Environmental Systems, San Francisco, California, July, 2008. 